\section{Visualising early lung disease in CF: the emergence of MRI}

\author{
Jane C Davies
}

The potential of magnetic resonance imaging (MRI) in lung imaging appears to be increasing year-on-year; ${ }^{1}$ having previously been considered a tool unlikely suitable for an air-filled organ, both hyperpolarised gas and more standard approaches are generating new interest within the field of cystic fibrosis (CF). The lack of radiation involved is an attractive feature, but are new outcome measures really needed, and if so, in which particular groups?

There is now widespread recognition that lung disease begins early in CF, but can remain undetected and therefore potentially inadequately treated. In particular, forced expiratory manoeuvres measured by spirometry lack sensitivity: alternative techniques such as multibreath washout-derived lung clearance index (LCI), high-resolution CT scanning and bronchoalveolar lavage assessment of inflammation are all abnormal in proportions of infants with completely normal spirometry. Evolving acceptance of this issue by regulatory agencies is evidenced by the recent approval of several paediatric drug trials based on these outcome measures (Clinicaltrials.gov: NCT02742519, NCT02950883). As the care of patients with CF improves, lung function may in fact remain well-preserved into later life. In older patients with normal spirometry, both $\mathrm{LCI}^{2}$ and hyperpolarised helium MRI $(\mathrm{HeMRI})^{3}$ are able to detect a

Correspondence to Dr Jane C. Davies, Gene Therapy, Imperial College, Emmanuel Kaye Building, Manresa Rd, London SW3 6LR, UK; j.c.davies@imperial.ac.uk significant response to the cystic fibrosis transmembrane conductance regulator (CFTR) potentiator, ivacaftor lending support to the use of such new agents even in subjects with apparently healthy lungs, who may be those with the greatest long-term potential to benefit. Thus, the case seems to be well made that outcome measures with enhanced sensitivity are urgently needed.

The study by Marshall et $a l^{4}$ was a head-to-head comparison of spirometry, LCI, CT and MRI (both ${ }^{3} \mathrm{He}$ and conventional) in children aged 6-16 years; patients with $\mathrm{CF}$ were stable with normal spirometry and were compared with agematched healthy controls. As expected, LCI and CT revealed changes in children with CF, but ${ }^{3} \mathrm{HeMRI}$ possessed the greatest sensitivity, being abnormal in all but two cases (89\%); in fact, these two had an atypical diagnosis and the R117H-7T mutation. Disease was quantified in one of two ways, $\%$ of unventilated lung and coefficient of variation (patchiness) of gas distribution, the former giving the greatest sensitivity and being well visualised on the examples presented.

So where does this study leave us? While the data contribute to the growing body of evidence supporting hyperpolarised gas MRI for lung imaging, the availability of the technique remains limited. The authors speculate that the use of ${ }^{129} \mathrm{Xe}$ could reduce costs and broaden the applicability of the test. In parallel, several teams are developing MRI techniques which are not dependent on hyperpolarised gas inhalation ${ }^{5}$ and which, should they become sufficiently discriminating, may provide an alternative approach. Taken together, these studies provide encouraging evidence that MRI for lung disease may be coming of age.

Competing interests None declared.

Provenance and peer review Commissioned; internally peer reviewed.

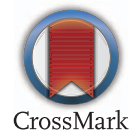

To cite Davies JC. Thorax 2017;72:682.

Published Online First 14 April 2017

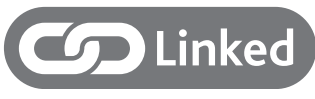

http://dx.doi.org/10.1136/thoraxjnl-2016-208948

Thorax 2017;72:682. doi:10.1136/

thoraxjnl-2017-210297

\section{REFERENCES}

1 Wielpütz MO, Mall MA. MRI accelerating progress in functional assessment of cystic fibrosis lung disease. J Cyst Fibros 2017;16:165-7.

2 Davies J, Sheridan H, Bell N, et al. Assessment of clinical response to ivacaftor with lung clearance index in cystic fibrosis patients with a G551D-CFTR mutation and preserved spirometry: a randomised controlled trial. Lancet Respir Med 2013;1:630-8.

3 Altes TA, Johnson M, Fidler $M$, et al. Use of hyperpolarized helium-3 MRI to assess response to ivacaftor treatment in patients with cystic fibrosis. J Cyst Fibros 2017;16:267-74.

4 Marshall H, Horsley A, Taylor CJ, et al. Detection of early subclinical lung disease in children with cystic fibrosis by lung ventilation imaging with hyperpolarised gas MRI. Thorax. Published Online First: 6 Mar 2017. doi:10.1136/thoraxjnl-2016208948.

2017:72:760-2.

5 Stahl M, Wielpütz MO, Graeber SY, et al. Comparison of lung clearance index and magnetic resonance imaging for assessment of lung disease in children with cystic fibrosis. Am J Respir Crit Care Med 2017;195:349-59. 